\title{
Quasi-static Process Modelling of Deep Cold Rolling on Ti-6Al-4V
}

\author{
A. Lim ${ }^{1,2, a^{*}}$, S. Castagne ${ }^{1}$, C.C. Wong ${ }^{2}$ \\ ${ }^{1}$ School of Mechanical and Aerospace Engineering, Nanyang Technological University, \\ Singapore \\ ${ }^{2}$ Advanced Technology Centre, Rolls-Royce Singapore Pte Ltd, Singapore \\ alims0200@e.ntu.edu.sg
}

\begin{abstract}
Keywords: Deep Cold Rolling, Residual Stress Modelling Methods, Residual Stresses in Manufacturing Process, Simulation, Abaqus
\end{abstract}

\begin{abstract}
This paper proposes a 3D Finite Element (FE) model for the deep cold rolling process, modelled using the commercially available Abaqus/Standard FE software, and used the afore mentioned model to study the effects of pressure and tool diameter on the residual stress profile predicted. The model was validated with experimental data. The predicted results showed about $20 \%$ deviation for the measured data in the near surface region, but showed good correlation at the compressive-tensile cross-over point. Reasons for this deviation are explored and analysed with several recommendations made to improve future FE models.
\end{abstract}

\section{Introduction}

Mechanical surface treatment (MST) methods physically alter the surface and near sub-surface regions of a component, creating a region of compressive residual stress. This compressive residual stress improves fatigue life and foreign object damage tolerance. Deep cold rolling (DCR) is a mechanical surface treatment and its main objective is to induce deep compressive residual stresses in the surface and sub-surface layers (up to $1 \mathrm{~mm}$ ) of the component [1].

While the high compressive stresses generated by DCR in the near surface layers can extend fatigue life, it is crucial that the location and magnitude of the corresponding balancing tensile residual stresses are carefully managed and understood, as they can negate the benefits of the near surface compressive residual stress and adversely affect the fatigue life of the component [2]. However, experimental determination of residual stress distributions by the hole drilling method and the X-ray diffraction method is time consuming, expensive and limited to discrete measurement points. Hence, FE simulation is a crucial tool in the process optimization of the DCR method as it allows the user to analyse and predict the residual stress profile of the component.

DCR creates different directional stress profiles and is reliant on both plastic deformation at the surface and Hertzian stress at the sub-surface region to generate the resultant residual stress profiles. Previous quasi-static finite element studies of the DCR process focused on the mechanics of a single pass [3]. While useful for understanding the mechanics of the DCR process, modelling of a single pass is insufficient to study the broader residual stress distributions.

The objective of this study is to propose and validate a FE model to simulate the DCR process. This model will enable a deeper understanding of the broader residual stress distributions caused by the DCR process.

\section{Methodology}

Material Model. The material model attributed to the model is isotropic elastic-plastic with strain hardening. A Young's modulus of $113.8 \mathrm{GPa}$ and a Poisson ratio of 0.342 are prescribed for the elastic portion of the material model. The strain hardening portion of the material model was 
experimentally determined via uniaxial compression of identical Ti-6Al-4V material in cylinder geometry measuring $6.75 \mathrm{~mm}$ height by $4.5 \mathrm{~mm}$ diameter. The machine stiffness is taken into account using plate to plate compression. Figure 1 shows the determined yield stress against plastic strain.

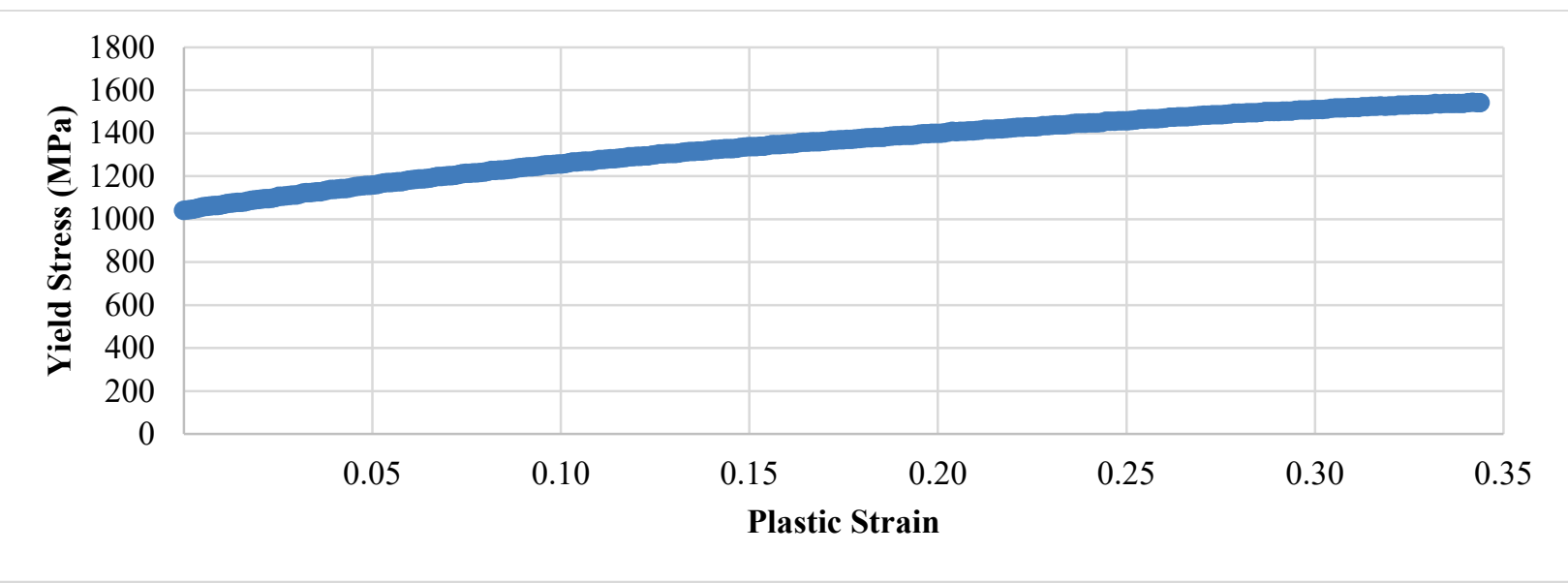

Figure 1: Yield stress against plastic strain for Ti-6Al-4V

Finite Element Model. The FE DCR model proposed is a 3-dimensional rectangular test coupon measuring $35 \mathrm{~mm}$ x $30 \mathrm{~mm}$ x $10 \mathrm{~mm}$. commercially available FE code Abaqus/Standard V6.14 was used to model the test coupon. The 3D deformable element type used was the C3D8R element, an 8node linear brick, using 56916 elements to model the test coupon. Uniform elements were used in the DCR zone to increase the resolution of the treated area, as shown in Figure 2a. Sensitivity analysis on the thickness of the test coupon was carried out to ensure that the thickness did not affect the residual stress profile generated.

To reduce computational time, a treated area of $15 \mathrm{~mm}$ lengthwise (LW - parallel to direction of rolling) by $10 \mathrm{~mm}$ crosswise ( $\mathrm{CW}$ - perpendicular to direction of rolling) was prescribed. The deep cold rolled zone allowed enough area for a strain gage rosette to be applied onto the experimental test block for experimental validation.

The DCR tool is made of tungsten carbide and has a Vickers hardness of approximately $2000 \mathrm{HV}$. Since this is several orders of magnitude harder than the test coupon, the tool was modelled as an analytical rigid surface. The centre node of the modelled tool was used as the main reference and control point. The equivalent concentrated force can be calculated using the following formula:

$$
F=\left[\frac{\pi}{4} * d_{\text {piston }}^{2} * P \cos (\theta)\right]
$$

where, $d_{\text {piston }}$ is the piston diameter, P is the applied pressure and $\theta$ is the angle of the tool to the normal.

The movement of the ball is controlled via translating the centre node of the ball, in the length and breadth direction. The DCR tool has an inner assembly containing the burnishing ball which is free floating (as shown in Figure 2b). The force prescribed determines the vertical position of the ball, relative to the component surface at equilibrium. Therefore, the displacement of the ball in the $\mathrm{Z}$ direction (normal to surface of the component) was left free. This is to mimic the operating mechanics of the DCR tool where the inner tool assembly is free to move in an up and down motion within the outer tool housing up to the limits of the stroke length. This allows the DCR tool to adapt to minor variations in geometry. 
The rotational degrees of freedom of the ball were also left free, ensuring that the ball is able to roll across the surface. The penalty formulation with a friction coefficient of 0.2 was used to model the surface to surface contact between the DCR ball and the work piece [4]. This also ensures that the ball rolls rather than slides across the component surface.

The boundary conditions were imposed such that the bottom surface of the test coupon is pinned. Since the test coupon is of sufficient thickness, it was assumed that the spring back of the coupon after treatment is negligible. The dimensions of the test coupon, the mesh, elements used and the boundary conditions are illustrated in Figure 2.

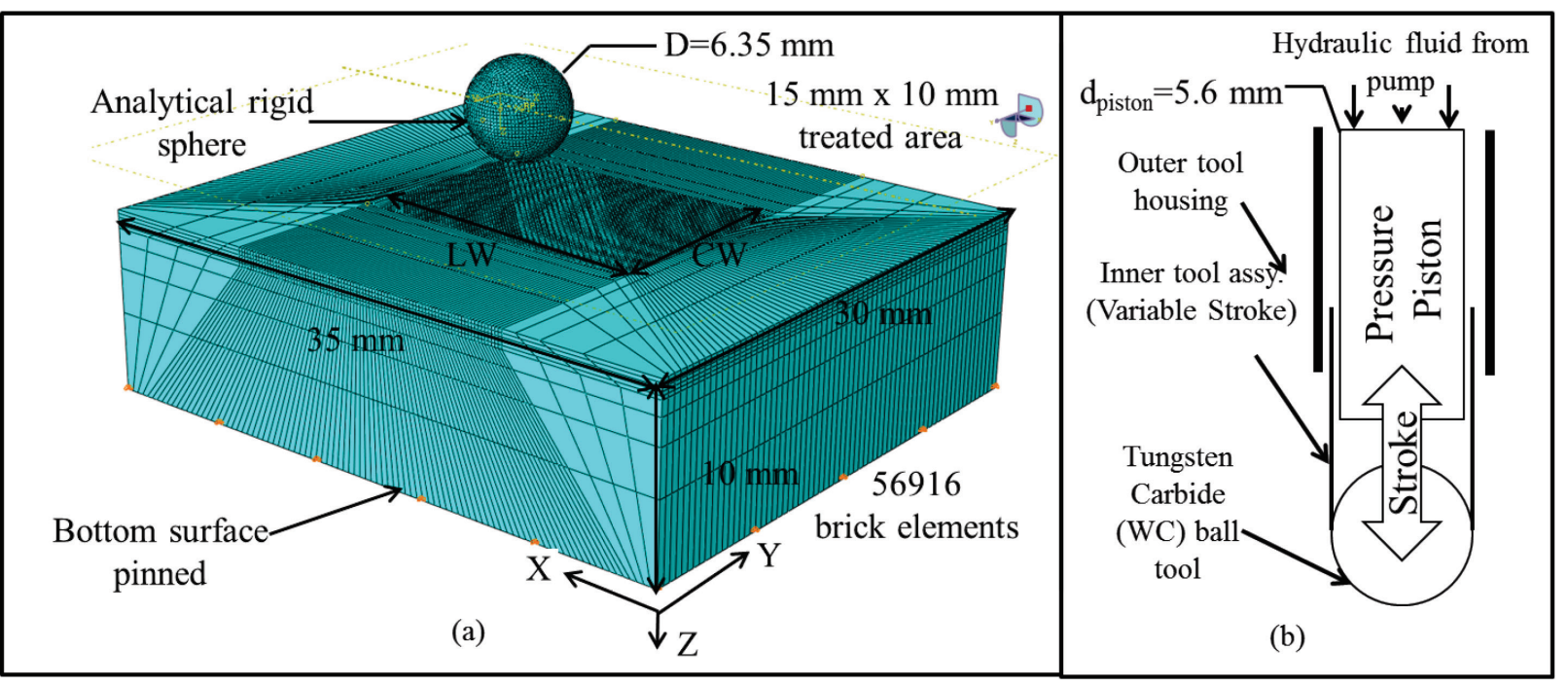

Figure 2: (a) Finite element model and set-up and (b) schematic of DCR tool

Experimental Setup and DCR Tool. Experiments were carried out to validate the proposed FE model. A flat rectangular test coupon of Ti-6Al-4V, measuring $35 \mathrm{~mm}$ × $30 \mathrm{~mm}$ x $20 \mathrm{~mm}$ was mounted on a work piece holding fixture and deep cold rolled. The DCR tool $(6.35 \mathrm{~mm}$ ball diameter), supplied by Ecoroll $\mathrm{AG}{ }^{\circledR}$, at $20.0 \mathrm{MPa}$ and $38.0 \mathrm{MPa}$ of pressure was used to perform the treatment. The test coupon was orientated such that the rolling direction of the DCR process is identical to the rolling direction that was used in the manufacturing of the test coupons. Figure 3 shows the DCR process experimental set up.

The residual stress profile of the test coupon was determined at the centre of the treated area using the central hole drilling method (CHD). The strain relaxation via the incremental drill method was measured and the residual stresses were back calculated as described in ASTM 837. A final hole depth of $1.4 \mathrm{~mm}$ was drilled in order to determine the stresses up to a depth of $1024 \mu \mathrm{m}$.

\section{Results and Discussion}

FE Model Validation. The finite element model was validated using experimental CHD data. In this study, the experimental and finite element data was normalized against the absolute maximum residual stress determined experimentally in the $\mathrm{CW}$ direction. The normalized graph is presented in Figure 4. 


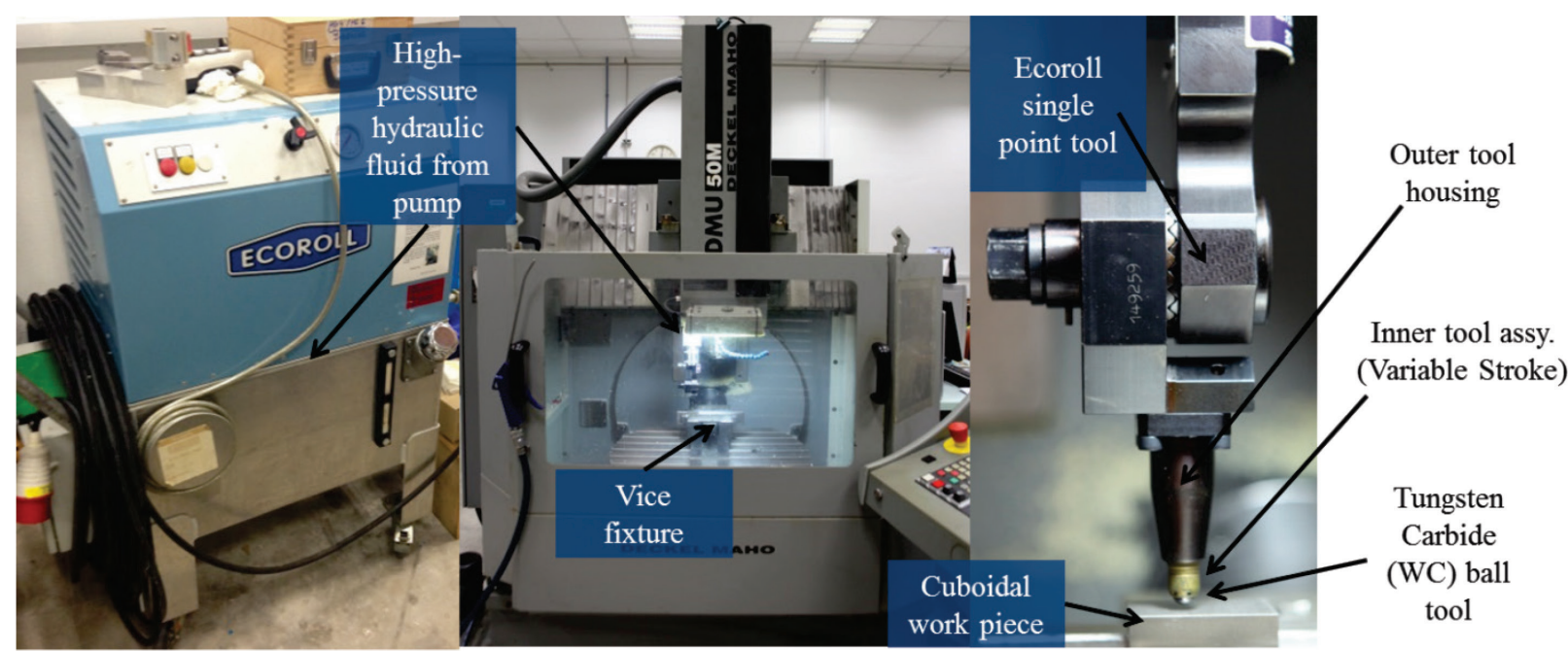

Figure 3: Experimental DCR set-up on a 3-axis CNC mill

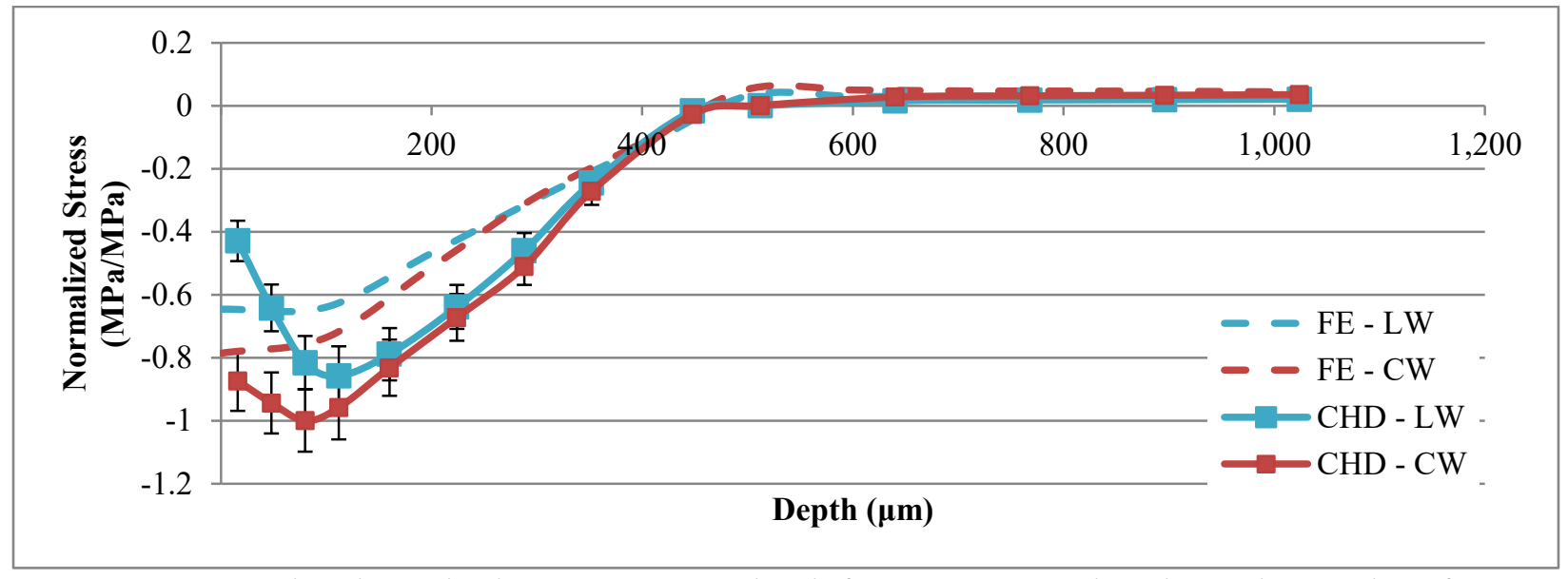

Figure 4: Normalized residual stress against depth for experimental and simulation data for 20.0 $M P a$

From Figure 4, it can be seen that there is some divergence of the residual stress in the near surface region. The FE model over-predicted the surface stress in the LW direction (parallel to rolling) at $-0.43 \mathrm{MPa} / \mathrm{MPa}$ measured against $-0.63 \mathrm{MPa} / \mathrm{MPa}$ predicted, and under predicted the surface stress in the $\mathrm{CW}$ direction (perpendicular to rolling) at $-0.87 \mathrm{MPa} / \mathrm{MPa}$ measured against $0.78 \mathrm{MPa} / \mathrm{MPa}$ predicted. Figure 5 shows the residual stress profiles when the pressure is increased to $38.0 \mathrm{MPa}$. There is a similar deviation between the measured and predicted residual stress profiles for both cases. 


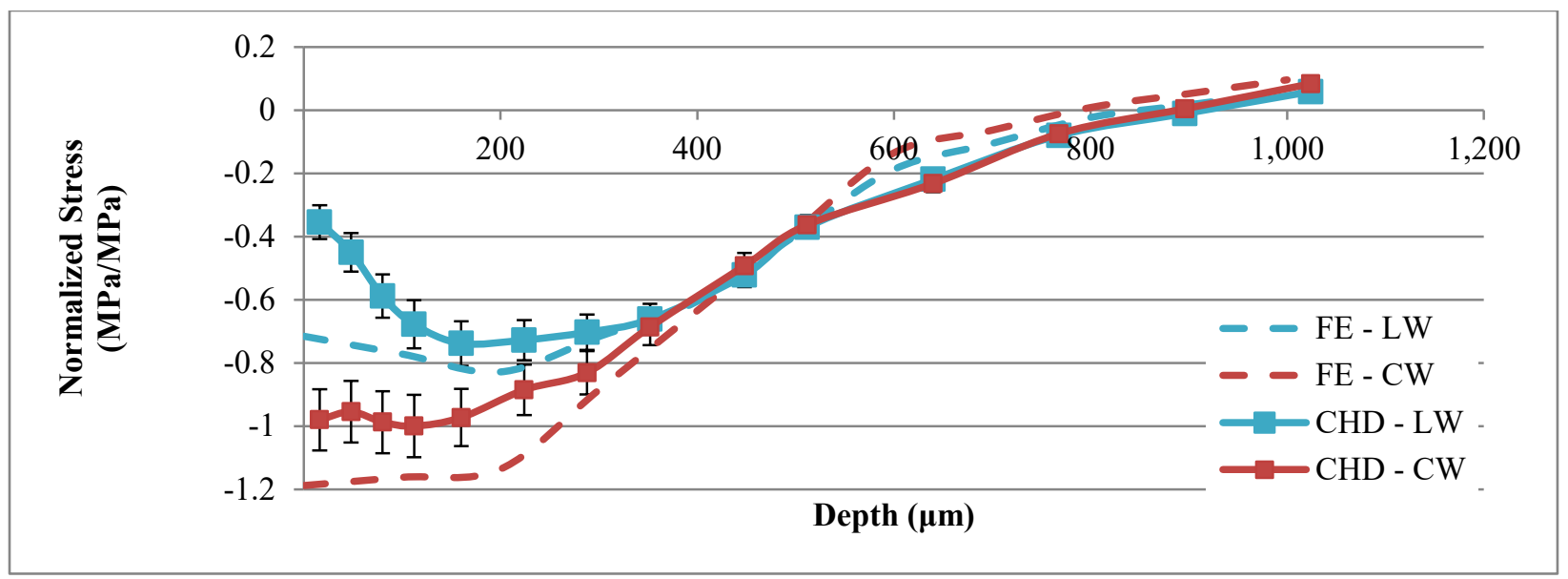

Figure 5: Normalized residual stress against depth for experimental and simulation data for 38.0 $\mathrm{MPa}$

Deeper into the material, both FE models are able to predict the general trend of the residual stress profile and the Compressive-Tensile Cross-Over point (CTCO). The CTCO point is of particular importance as the location of the balancing tensile stress needs to be able to be understood and predicted.

Regarding the deviation between the FE and CHD data, the material model used assumed that the material was isotropic. Further compression tests taking into account the rolling direction of the manufacturing process should be carried out to determine if this simplifying assumption holds valid. A more detailed model, such as the Johnson-Cook model, could be considered for future FE simulations.

In this study, the implicit FE code is used and it does not take time into consideration when performing iterations. The time step used in the code relates to the relative position of the components and forces applied rather than actual time as a parameter. The implicit code does not take strain rate into consideration

Another potential cause of the deviation could be the measurement method used to determine the experimental residual stress profiles. While CHD is an affordable and quick way of determining residual stress depth profiles, it has limited resolution in the near surface region (up to about 200 $\mu \mathrm{m})$. This can be seen in the error bars shown in Figure 4 for the experimental graphs. At the near surface region, CHD has an error of about $10-15 \% \sigma_{\max }$. X-ray diffraction (XRD) or Focused Ion Beam (FIB) methods could potentially be used to attain more accurate data in the near surface region.

A final source of error that may have resulted in the deviation is the concentrated force applied based on equation (1). The equation calculates the theoretical force exerted by the DCR ball element on the component based on the pressure applied and the projected area of the pressure piston. A dynamometer could be placed under the component fixture to determine the actual force applied on the test coupon. This measured force can then be used to further improve the FE model.

The motivation behind process modelling the DCR process can be seen in the stress plots of the component shown in Figure 6. There is a zone of tensile residual stress that was formed near the boundary region at $1.0 \mathrm{~mm}$ in depth for the $38.0 \mathrm{MPa}$ case that was not present for the $20.0 \mathrm{MPa}$ case. Knowledge of this zone of tensile stress will assist the process engineer in designing of the DCR zone and ensure that the component does not prematurely fail due to the tensile stress. 


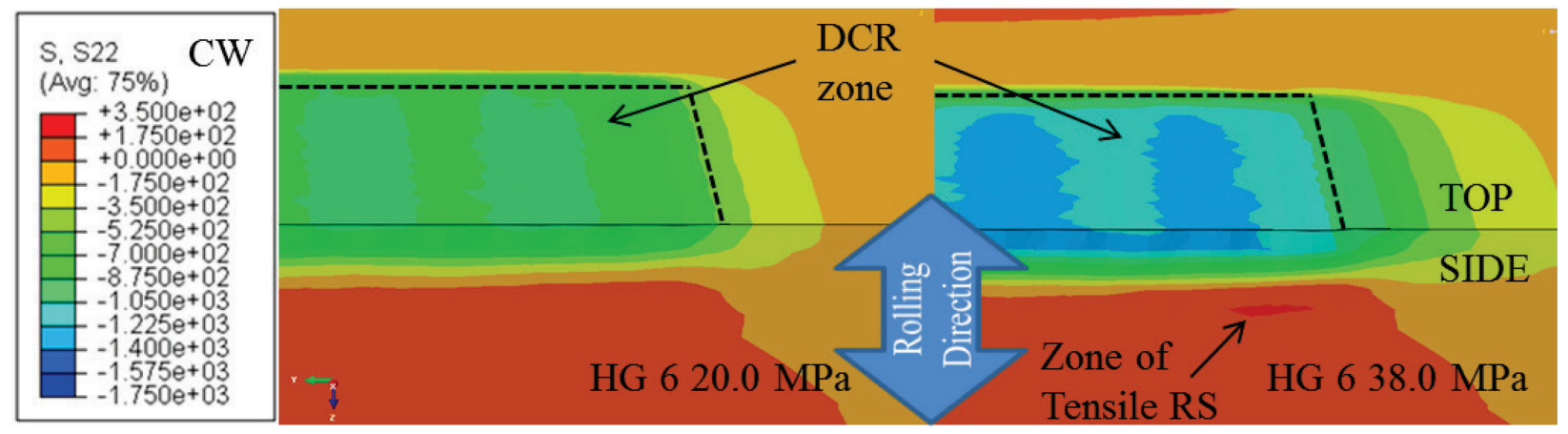

Figure 6: FE stress plot for $H G$ 6, 20.0 MPa and $38.0 \mathrm{MPa}$, cut at $x=17.5 \mathrm{~cm}$ (centre of treated zone)

\section{Conclusion}

This paper proposed a 3D FE model to simulate the DCR process. There is a maximum deviation of $0.2 \mathrm{MPa} / \mathrm{MPa}$ at the surface region between the predicted FE results and the CHD measured data. There is good agreement between the FE and CHD data beyond $400 \mu \mathrm{m}$. Some causes of the deviation between the FE predicted values and the experimental data have been proposed and will need to be verified. Different material models will be studied in order to better understand the effects of the material models on the accuracy of the FE simulation.

\section{Acknowledgement}

This study was conducted using funding from the Singapore Economic Development Board (EDB) and Rolls-Royce Singapore Pte. Ltd. Joint Industrial Post-graduate Programme (IPP) grant. Finite element modelling for this study was conducted in Computer Aided Engineering Laboratory 1 of School of Mechanical and Aerospace Engineering, Nanyang Technological University.

\section{References}

[1] Altenberger, I. Deep rolling - the past, the present and the future. in Proceedings of 9th International Conference on Shot Peening, Sept. 2005.

[2] Webster, G. and A. Ezeilo, Residual stress distributions and their influence on fatigue lifetimes. International Journal of Fatigue, 2001. 23: p. 375-383.

http://dx.doi.org/10.1016/S0142-1123(01)00133-5

[3] Balland, P., et al., Mechanics of the burnishing process. Precision Engineering, 2013. 37(1): p. 129-134.

http://dx.doi.org/10.1016/j.precisioneng.2012.07.008

[4] Lim, A., S. Castagne, and C.C. Wong, Effect of Friction Coefficient on Finite Element Modeling of the Deep Cold Rolling Process, in Shot Peening: $12^{\text {th }}$ International Conference, ICSP 12, Goslar, Germany, September 2014, Proceedings, L. Wagner, Editor. 2014: ClausthalZellerfeld, Germany. p. 376-380. 